\title{
WAVELET BASED MEDICAL IMAGE CONTRAST AND RESOLUTION ENHANCEMENT BY INCORPORATING CUCKOO SEARCH ALGORITHM AND SVD
}

\author{
Sandeepa K S ${ }^{1, *}$, B.N. Jagadale ${ }^{1}$, J.S.Bhat ${ }^{2}$ \\ ${ }^{1}$ Department of Electronics, Kuvempu University, 577451, Karnataka, India. \\ ${ }^{2}$ Department of Physics, Karnataka University Dharwad 580003, Karnataka, India \\ *Sandeepa K S: $\underline{\text { saan.ks@gmail.com }}$
}

\begin{abstract}
A new approach based on Discrete Wavelet Transform (DWT) and Singular Value Decomposition (SVD) for medical image contrast enhancement is presented. The contrast of the input image is enhanced by proposed fusion technique between wavelet based masking using Cuckoo Search Algorithm (CSA) and mask based singular value decomposition. Resolution is enhanced by combining interpolated high frequency sub band and maximum value fused low frequency sub band using mask technique. Experimental results are tested by measuring Peak Signal to Noise Ratio (PSNR) with other contrast and resolution enhancement techniques. Structural Similarity Index of Measure (SSIM) and Correlation coefficient is also evaluated to encourage the proposed method.
\end{abstract}

Keywords: DWT, Cuckoo search algorithm, SVD, Masking, Fusion, IDWT.

\section{Introduction}

The process of improving the image quality without knowing its source of degradation is called image enhancement. The image quality is improved by de-noising process, contrast improvement, preserving brightness, increasing resolution and so on. Image enhancement is one of the growing research fields with having existence in many applications. Satellite images processing, geographic image processing and medical image processing are the some of the areas where image enhancement techniques are used prominently. The contrast and resolution are the key issues in enhancing the digital image. The resolution is improved by interpolation techniques (i.e. the nearest neighbor, bilinear, and bi-cubic) and preserving the edges. A mathematical tool, Discrete Wavelet transforms (DWT) has been used in many applications ${ }^{(1-4)}$.

In medical image analysis, contrast enhancement helps to improve the clarity of diagnosis. Contrast enhancement is classified as example based and intensity based (5-6) enhancements. Intensity based enhancement is again classified as histogram based, transform domain, filtered based and masking based approaches. The field of Histogram Equalization (HE) has Generalized Histogram Equalization (GHE), Bi-histogram, Brightness Preserving Bi-Histogram Equalization, Dualistic Sub Image Histogram Equalization (DSIHE), Recursive Sub Image Histogram Equalization (RSIHE), Adaptive Histogram, Automatic Histogram, Selective dynamic histogram Equalization; Threshold optimized histogram equalization ${ }^{(7-14)}$. Masking based contrast enhancement with some modification is documented in various papers ${ }^{(15-16)}$. Recent approach to enhancement of image has done by Un-Sharpe masking technique, where scale value is used for masking process (17-18). Image contrast and brightness enhancement using cuckoo search algorithm based DWT-SVD method ${ }^{(19-22)}$ have been tried in recent years.

This paper, introduces new method DWT-CS-mask SVD to give better contrast and resolution by preserving edge information.

The paper structured as follows. Section 2 presents the proposed method. Section 3 discusses result and discussion and section 4 gives the conclusion of the algorithm. 


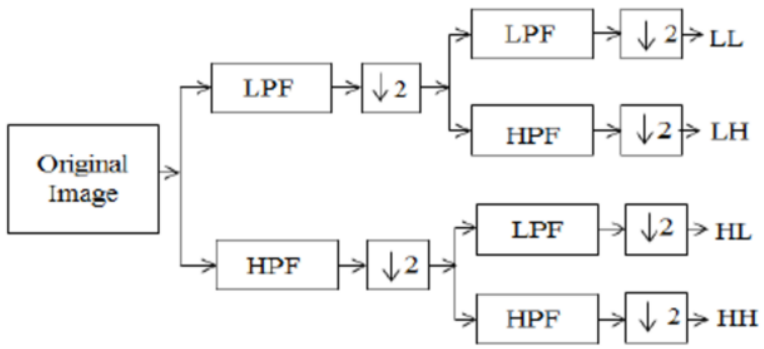

Fig. 1. Block diagram of DWT filter banks of level 1.

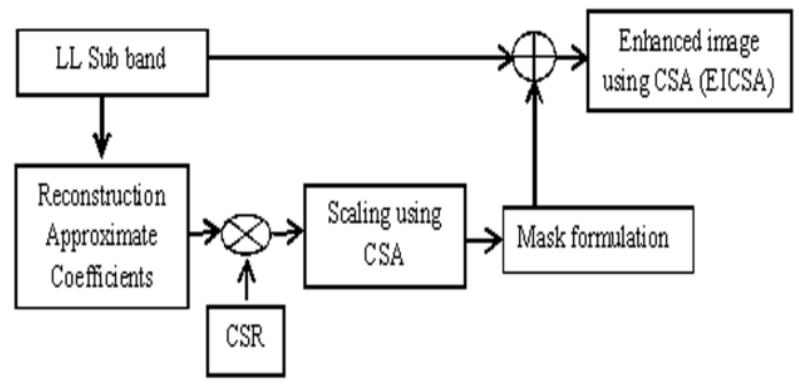

Fig. 2. Block diagram of EICSA method.

\section{Proposed method}

\subsection{Image Decomposition}

Most of the image processing applications use wavelet transforms because of their inherent properties. It decomposes the image into frequency sub-bands namely LL, LH, HL and HH bands as shown in figure 1. The LL sub band contains illumination information and other sub bands are preserving edge and high frequency information.

\subsection{Enhanced Image using Cuckoo Search Algorithm (EICSA)}

The contrast of the image is improved by wavelet based unsharp masking technique ${ }^{(23)}$, in which masking formulation obtained by subtracting the scaled reconstructed approximate coefficient and reconstructed low pass signal. The decomposed approximation coefficients are obtained from equation (1) and reconstructed approximate coefficients are obtained using Inverse Discrete Wavelet Transformation (IDWT) as given by Equation (2).

$$
\begin{aligned}
& W_{\varphi}\left(j_{0}, m, n\right)=\frac{1}{\sqrt{M N}} \sum_{x=0}^{M-1} \sum_{y=0}^{N-1} f(x, y) \varphi_{j_{0}, m, n}(x, y) \\
& f_{(x, y)}^{A}=\frac{1}{\sqrt{M N}} \sum_{m} \sum_{n} W_{\varphi}\left(j_{0}, m, n\right) \varphi_{j_{0}, m, n}(x, y)
\end{aligned}
$$

where, $W_{\varphi}\left(j_{0}, m, n\right)$ is the approximation coefficient,

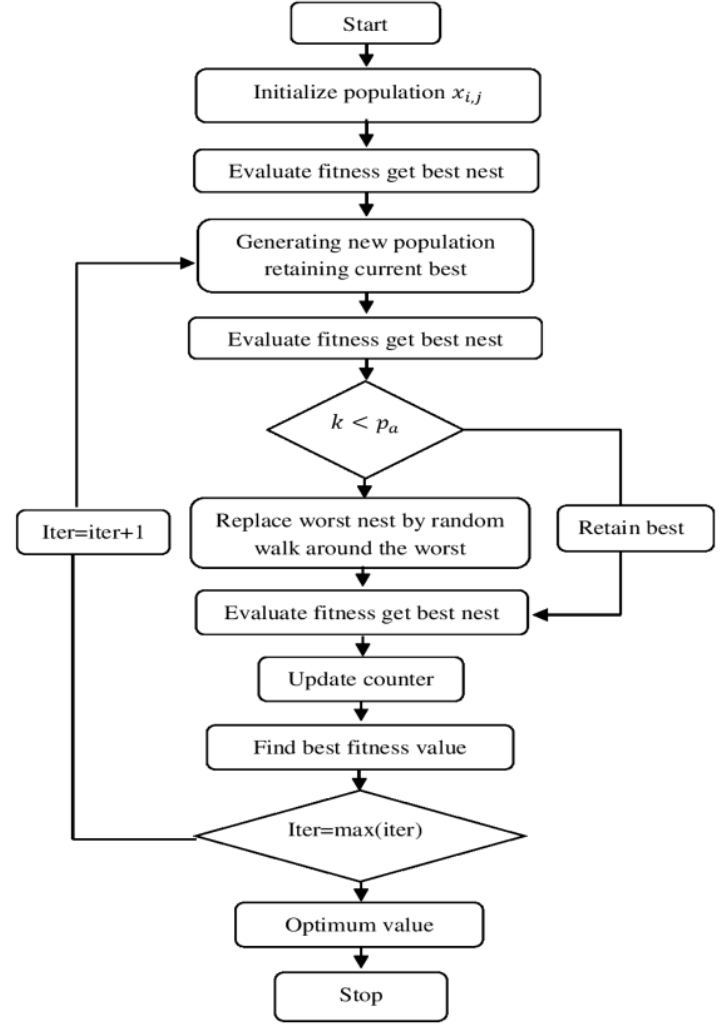

Fig. 3. Block diagram of EICSA method.

$f(x, y)$ is the input time domain image with discrete variable $\mathrm{x}, \mathrm{y}$ with the size $\mathrm{M} \mathrm{x} \mathrm{N} . \varphi_{j_{0}, m, n}(x, y)$ is the scale function and $f_{(x, y)}^{A}$ is the reconstructed approximation coefficient ${ }^{(24)}$.

The reconstructed approximation coefficient is scaled using a cuckoo search algorithm and mask is formulated. Wavelet mask is added with original image and obtained output image considered as Enhanced Image using Cuckoo Search Algorithm (EICSA) as shown in the figure 2.

Cuckoo Search (CS) algorithm is meta-heuristic optimization algorithm based on captivating reproduction policy of the bird cuckoo developed by Yang and Deb ${ }^{(25)}$. Cuckoo lays their eggs in the host bird nest, it doesn't construct nest. If host bird identifies cuckoo egg, it will either rebuilds their nest or they will abandon their eggs.

The CS is based on three idealized rules:

a. At a time, each cuckoo lays one egg in a randomly chosen nest.

b. Next generation will carry over by best nests with high quality of eggs (solutions). 
c. The number of available host nests is fixed and a host can discover an alien egg with probability 'pa' $\varepsilon\left[\begin{array}{ll}0 & 1\end{array}\right]$.

The host birds either abandon the nest or throw the egg away. This implementation is carried by replacing 'pa'. Each egg corresponds to a solution and its fitness is calculated. A new solution generated using Levy flight as given in the equation 3 .

$$
x_{i}(t+1)=x_{i}(t)+\alpha \otimes \operatorname{levy}(\lambda)
$$

where, $\alpha$ is step size. It essentially provides a random walk while random step length is drawn from a Levy distribution as given below

$$
\operatorname{levy}(\lambda)=t^{-\lambda}: 1<\lambda \leq 3
$$

The iterative process continues up to replacement of a not good egg and finding optimum solution ${ }^{(26)}$. The flow chart of cuckoo search algorithm is shown in the figure 3.

\subsection{Enhancing Image using SVD (EISVD)}

SVD based masking technique proposed in which masking formulation obtained by subtracting inverse singular value decomposition and EICSA image. SVD mask is added with original image and enhanced Image using SVD (EISVD) is obtained as shown in figure 4 .

To get inverse singular value decomposition, the original LR image and EICSA image are decomposed by SVD as given in equations (5) and (6).

$$
\begin{aligned}
& I 1=U 1 \times S 1 \times V 1 \\
& I 2=U 2 \times S 2 \times V 2
\end{aligned}
$$

Here U1, V1, U2 and V2 are the orthogonal matrices. S1 and S2 are diagonal matrices containing intensity

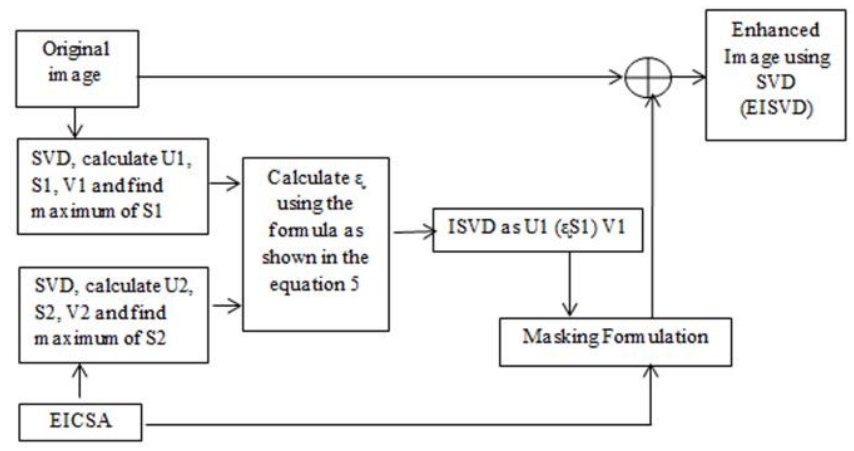

Fig. 4. Block diagram of EISVD method

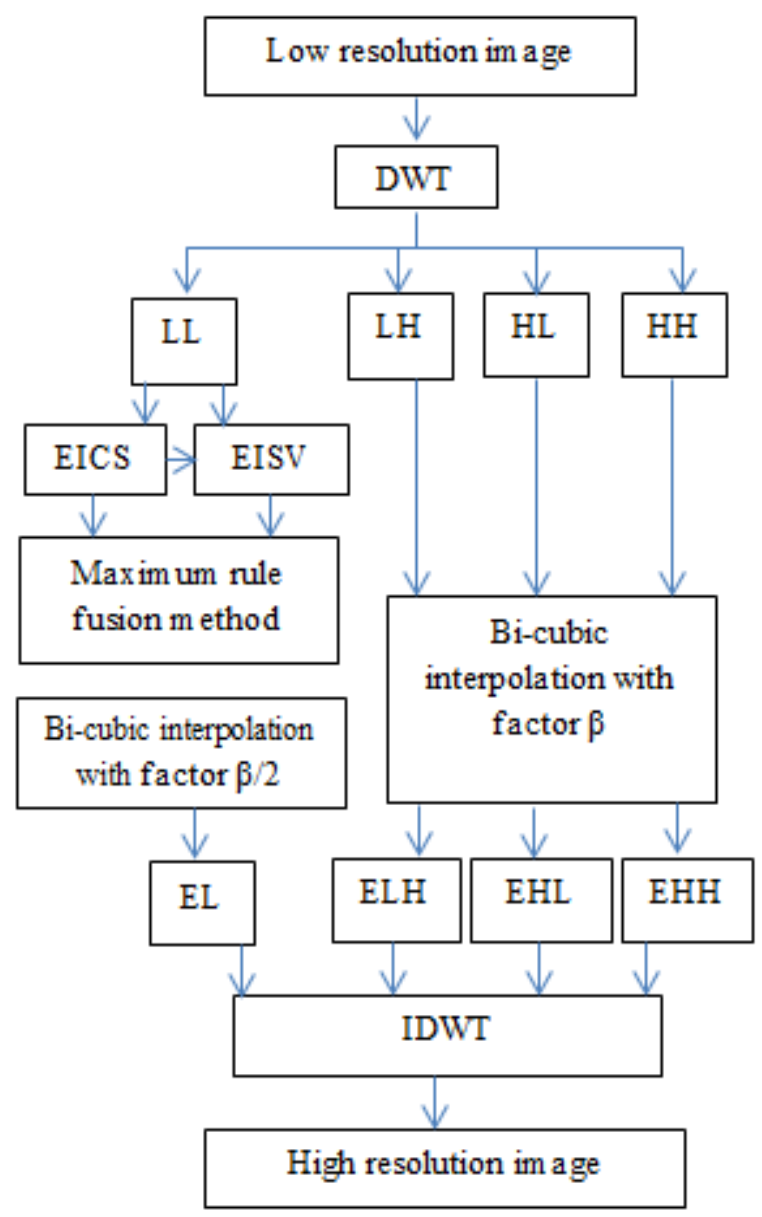

Fig. 5. Block diagram of proposed method

information. To enhance the image $\varepsilon$ is calculated by LR and EICSA images as given in equation (7).

$$
\varepsilon=\frac{\max (S 1)+\max (S 2)}{2 X \max (S 1)}
$$

New image with improved contrast obtained from inverse singular value decomposition [27] as given in equation (8).

$$
N I=U 1 \times \varepsilon S 1 \times V 2
$$

\subsection{Interpolation and inverse process}

The resolution of the image is improved by interpolating the obtained sub bands of the image. The high frequency sub bands are interpolated using the factor $\beta$ and image is obtained through max value based fusion of the EICSA and EISVD image, and is interpolated with factor $\beta / 2$. After applying bi-cubic interpolation to the intermediate stage, estimated sub bands are created, and then high resolution output image is obtained by applying IDWT, as illustrated in figure. 5. The output image got improved contrast and preserves more high frequency information than the image obtained by direct scaling of reconstructed approximations 
and SVD method.

\section{Results and discussion}

In this section, experimental results are discussed, and compared with the results obtained by using various methods for medical and ordinary images.

1. Qualitative and quantitative analysis of proposed method with medical image

2. Qualitative and quantitative analysis of proposed method with ordinary image.

\subsection{Medical image qualitative and quantitative analysis}

The performance of the proposed method is analysed using images of brain MR, face XR, MRI and ribs. Figure 6 shows original image, reconstructed approximation matrix, EICSA, EISVD and output image. The LL sub band of the original low resolution image is reconstructed as wavelet based low pass filtered image using equation 2. The EICSA and EISVD images are obtained by masking formulation; both the images are having acceptable visual information and its contrast also enhanced. The image fusion has done

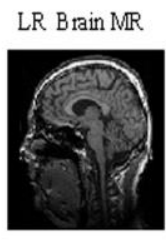

EISVD image

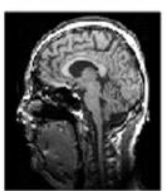

Reconstructed approximation image

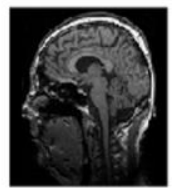

Fusion image

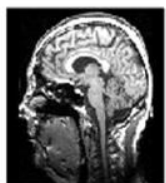

EICSA image

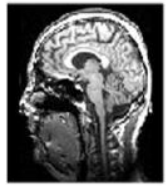

HR output image

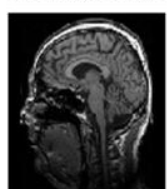

Fig. 6. Processed Brain MRI image in different stages
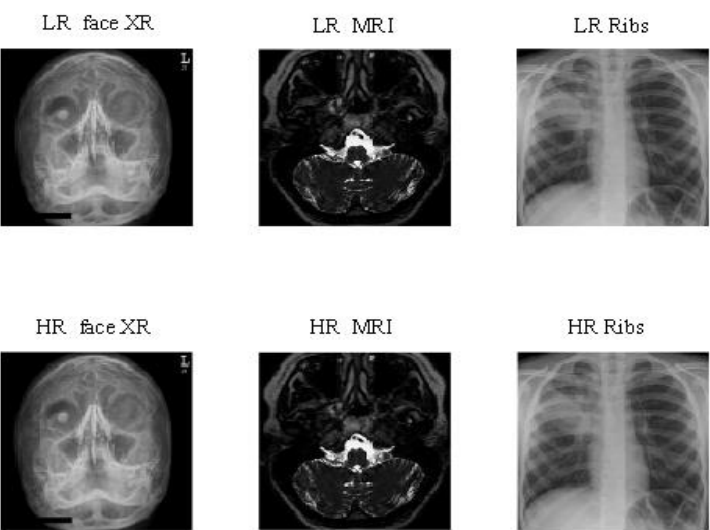

HR MRI

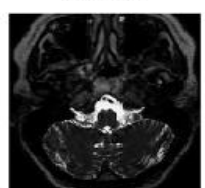

HR Ribs

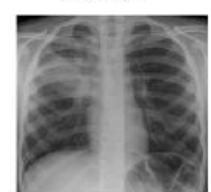

Fig. 7. Processed face XR, MRI, Ribs images by considering maximum value from both of the EICSA and EISVD images. The resultant image is contrast enhanced image. The bi-cubic interpolation method is applied to all sub bands and it makes the entire DWT sub bands to same size, high resolution image obtained by IDWT process. The output image provides enhanced contrast and resolution than the original image. In figure 7 , original and enhanced image of the face XR, MRI and ribs are shown.

The quantitative analysis is carried out by comparing the results with existing methods in terms of PSNR ${ }^{(27)}$ and is given in the figure 9. The result of PSNR shows that, proposed method shows improvements over the previous work.

Table 2 contains SSIM value, Correlation coefficient and AMBE values show the performance of the proposed algorithm for contrast enhancement.
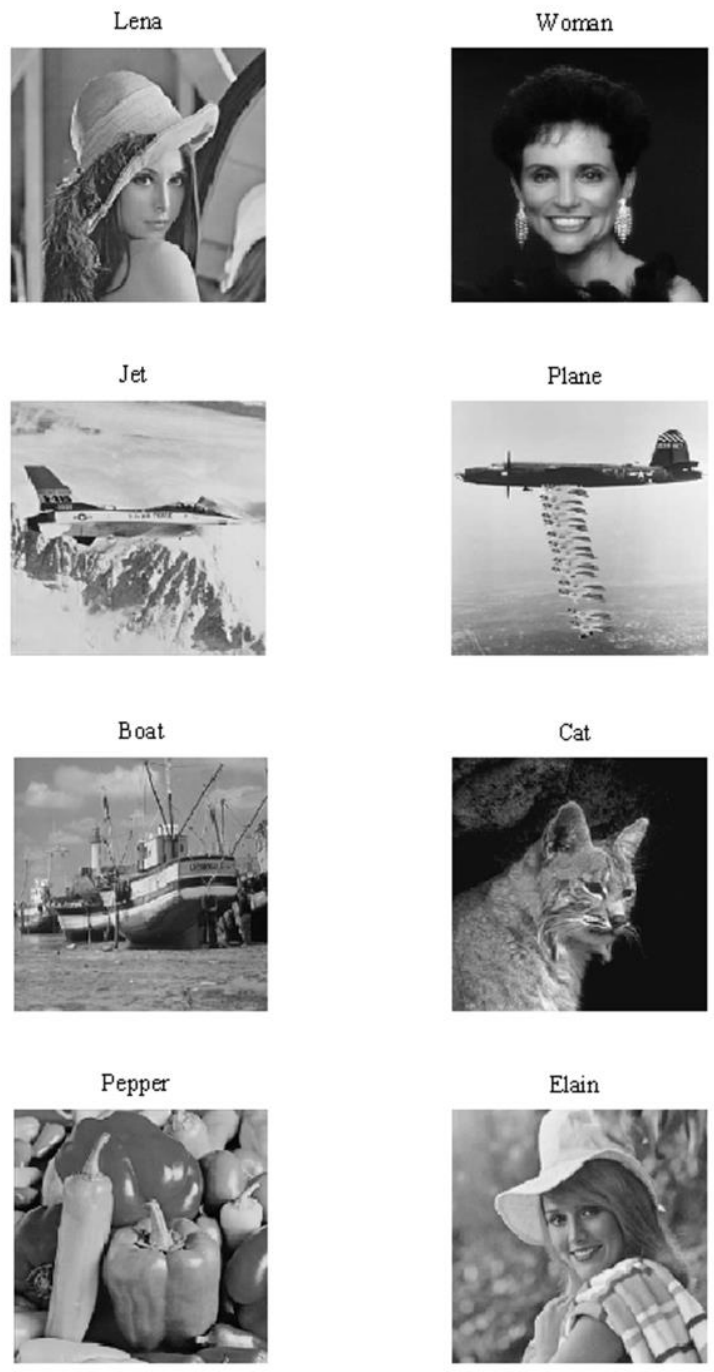

Fig. 8. Images of the ordinary tested images 


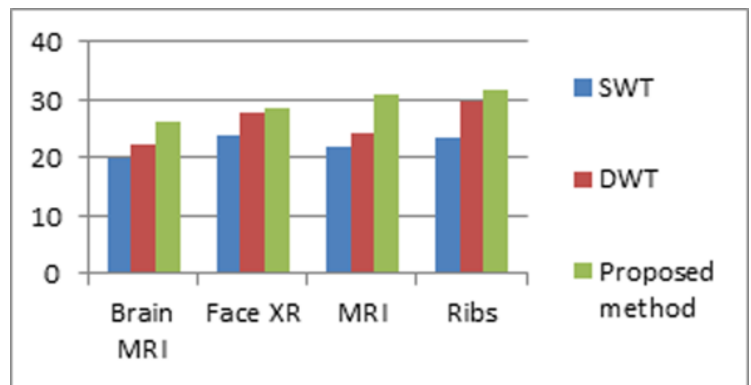

Fig. 9. PSNR comparisons

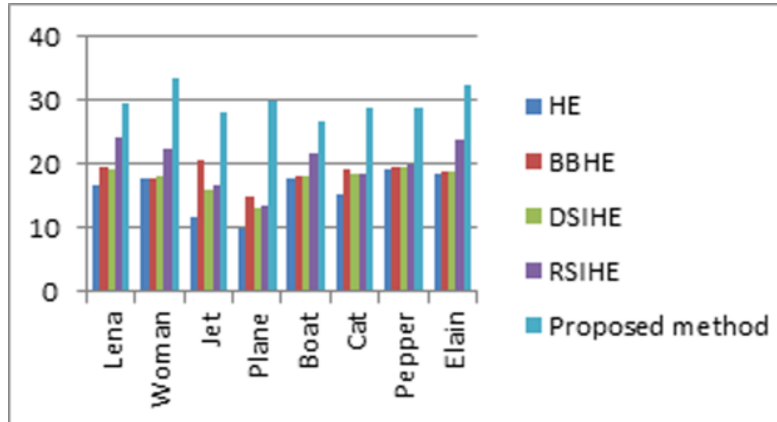

Fig. 10. PSNR comparisons of ordinary tested images

\subsection{Qualitative and quantitative analysis of ordinary images}

The proposed algorithm is tested for different format of ordinary images, Lena, Woman, Jet Plane, Boat, Cat and Pepper. The qualitative analyses of the enhanced images are shown in the figure 8 . In addition to qualitative evaluation, proposed method compared with various techniques like HE, BBHE, DSIHE, RSIHE ${ }^{(28)}$ using PSNR measurement are shown in the figure 10 .

The list of SSIM, correlation coefficient values and AMBE values to encourage the performance of the proposed method are given in Table 3.

\subsection{Figures and tables}

Table 1: Parameter used in the cuckoo search algorithm

\begin{tabular}{|c|c|}
\hline Parameters & Value \\
\hline Number of Nest & 50 \\
\hline Alien egg discover rate pa & 0.25 \\
\hline Number of iteration & 50 \\
\hline Lower bound value & 0 \\
\hline Upper bound value & 1 \\
\hline Dimension & 1 \\
\hline
\end{tabular}

\section{Conclusions}

In this paper, wavelet based contrast and resolution
Table 2: SSIM, Correlation coefficient and AMBE of medical

\begin{tabular}{|c|c|c|c|}
\multicolumn{5}{|c|}{ image } \\
\hline Images & SSIM & CC & AMBE \\
\hline Brain MRI & 0.9152 & 0.9705 & 0.0793 \\
\hline Face XR & 0.9489 & 0.9877 & 0.0583 \\
\hline MRI & 0.9349 & 0.9853 & 0.0202 \\
\hline Ribs & 0.9574 & 0.9870 & 0.0271 \\
\hline
\end{tabular}

Table 3: SSIM, Correlation coefficient and AMBE of the ordinary image

\begin{tabular}{|c|c|c|l|}
\hline Images & SSIM & CC & AMBE \\
\hline Lena & 0.8504 & 0.9844 & 1.1562 \\
\hline Woman & 0.9246 & 0.9974 & 1.3251 \\
\hline Jet & 0.8749 & 0.9748 & 0.0375 \\
\hline Plane & 0.9244 & 0.9867 & 0.5435 \\
\hline Boat & 0.7616 & 0.9671 & 0.0291 \\
\hline Cat & 0.8105 & 0.9870 & 1.8120 \\
\hline Pepper & 0.8242 & 0.9858 & 0.4021 \\
\hline Elain & 0.8575 & 0.9911 & 1.2318 \\
\hline
\end{tabular}

enhancement technique is proposed. For contrast improvement cuckoo search algorithm is used to get optimum scale value and SVD method is used to improve intensity of the image, both are followed by masking technique to obtain overall enhanced image. Maximum contrast enhancement achieved by applying maximum value fusion technique and resolution improved by applying interpolation method. The performance of the algorithm was studied by processing medical and ordinary image, experimental study of the PSNR, SSIM, AMBE and correlation coefficient values show that proposed method generates good quality visual outputs and higher performance.

\section{Acknowledgment}

The research work is supported by UGC -MRP, New Delhi, India.

\section{References}

(1) Y.Piao, I.Shin, and H.W.Park,"Image resolution enhancement using inter subband correlation in wavelet domain," in Proc. Int.Conf. Image Process, 2007, vol. 1, pp. I-445-448.

(2) H. Demirel and G. Anbarjafari, "Satellite image resolution enhancement using complex wavelet transform,"IEEE Geoscience and Remote Sensing Letter, vol. 7, no. 1, pp. 123-126, Jan. 2010. 
(3) S. Mallat, A Wavelet Tour of Signal Processing, 2nd ed. New York: Academic, 1999.

(4) Hasan Demirel and Gholamreza Anbarjafari," Discrete Wavelet Transform-Based Satellite Image Resolution Enhancement", IEEE TRANSACTIONS ON GEOSCIENCE AND REMOTE SENSING, VOL. 49, NO. 6, JUNE 2011

(5) B.Li.W.Xie, "Adaptive fractional differential approach and its application to medical image enhancement", comput. Electr.Eng.(2015) 324-335.

(6) A.F.D. Araujo,et all., "New artificial life model for image enhancement, Expert syst.Appl.41(2014) 58925906.

(7) H.Demirel, G. Anbarjafari, M.N.S. Jahromi, Image Equalization based on singular value decomposition,in:IEEE international symposium on computer and information sciences, October 2729,2008 pp 1-5.

(8) Yeong-taeg-kim Contrast enhancement using brightness preserving bi-histogram equalization",IEEE Transactions on Consumer Electronics (Volume: 43, Issue: 1, Feb 1997).

(9) Yu wang, Quin chen,Baeomin Zang,"Image enhancement based on equal area dualistic sub-image histogram equalization method", IEEE Transactions on Consumer Electronics ( Volume: 45, Issue: 1, Feb 1999 ).

(10) Kok swee Sim, C. P. Tso.," Recursive sub-image histogram equalization applied to gray scale images", Pattern Recognition Letters 28(10):1209-1221 · July 2007.

(11)C.Zuo,Q.Chen,X.sui, Range limited bi-histogram equalization for image contrast enhancement, optik 124(2013) 425-431.

(12) S.C. Huang,C.H.yeh, Image contrast enhancement for preserving mean brightness without losing features, Eng.appl.artif.intell.26(2013) 1487-1492.

(13) P.Shanmuga vadivu, K. Balasubramani," Thresholded and Optimized Histogram Equalization for contrast enhancement of images", Computers \& Electrical Engineering, Volume 40, Issue 3, April 2014, Pages 757-768.

(14) M.F. Khan, E Khan, ZA Abbasi," Segment selective dynamic histogram equalization for brightness preserving contrast enhancement of images", OptikInternational Journal for Light and ..., 2014 - Elsevier.

(15) Ching Chung Yang "A modification for the maskfiltering approach by superposing anisotropic derivatives in an image", Optik - International Journal for Light and Electron Optics Volume 122, Issue 18, September 2011, Pages 1684-1687.

(16) Ching Chung Yang "Color image enhancement by a modified mask-filtering approach", Optik International Journal for Light and Electron Optics Volume 123, Issue 19, October 2012, Pages 17651767.

(17) Guang Deng," A Generalized Unsharp Masking Algorithm", IEEE TRANSACTIONS ON IMAGE PROCESSING, VOL. 20, NO. 5, MAY 2011.

(18) A. Polesel, G.Ramponi,V.J.Mathews "Image enhancement via adaptive unsharp masking”, IEEE Transactions on Image Processing (Volume: 9, Issue: 3, Mar 2000 ).

(19) A.K. Bhandaria, V. Sonia, A. Kumara,n, G.K. Singhb, Cuckoo search algorithm based satellite image contrast and brightness enhancement using DWT-SVD, ISA Transactions.53 (2014) 1286-1296.

(20) Hasan Demirel, Cagri Ozcinar, and Gholamreza Anbarjafari," Satellite Image Contrast Enhancement Using Discrete Wavelet Transform and Singular Value Decomposition", IEEE GEOSCIENCE AND REMOTE SENSING LETTERS (2009).

(21) Bhandari AK, Kumar A, Padhy PK. Enhancement of low contrast satellite images using discrete cosine transform and singular value decomposition. World Acad Sci Eng Technol 2011;79:35-41.

(22) Y Yang,Z.Su,L.Sun. "medical image enhancement based on wavelet transform", Volume 46, Issue 2, 21 January 2010, p. 120 - 121,IET.

(23) E Daniel,J Anitha, “Optimum wavelet based masking for contrast enhancement of medical images using enhanced cuckoo search algorithm" Elsevier, computers in biology and medicine 71 (2016) 419-155.

(24) R.C. Gonzalez, R.E. Woods, Digital image processing, third ed., Pearson, India, 2014.

(25) X.S.Yang,S.Deb, “Cuckoo Search via levy flights” in: proceedings of world congress on nature and biological inspired computing (NaBIC), IEEE publications, 2009,pp,210-214.

(26) Shilpha Suresh, Dr.Shyam Lal, “An Eficient Cuckoo Search Algorithm based Multilevel Thresholding for Segmentation of Satellite Images Using Different Objective Functions", Expert Systems with Applications $58 \cdot$ April 2016,184-209.

(27) MZ Iqbal, A Ghafoor, AM Siddiqui, MM Riaz, U Khalid, Dual-tree complex wavelet transform and SVD 
based medical image resolution enhancement, Signal Processing ,Volume 105, December 2014, Pages 430437.

(28) Shin-Chia Huang, Chien-Hui Yeh,'Image contrast enhancement for preserving mean brightness without losing image features", Engineering Application of Artificial Intelligence 26 (2013) 1487-1492. 